Research paper

\title{
Virulence potential of commensal multidrug resistant Escherichia coli isolated from poultry in Brazil
}

\author{
Joseane Cristina Ferreira $^{\mathrm{a}}$, Rafael Antonio Casarin Penha Filho ${ }^{\mathrm{b}}$, Ana Paula Yorika Kuaye ${ }^{\mathrm{a}}$, \\ Leonardo Neves Andrade ${ }^{\mathrm{a}}$, Yung-Fu Chang ${ }^{\mathrm{c}}$, Ana Lúcia Costa Darini ${ }^{\mathrm{a}, *}$ \\ ${ }^{\text {a }}$ School of Pharmaceutical Sciences of Ribeirão Preto, University of São Paulo (USP), Ribeirão Preto, SP 14040-903, Brazil \\ ${ }^{\mathrm{b}}$ School of Agricultural and Veterinary Sciences, São Paulo State University (UNESP), Jaboticabal, SP 14884-900, Brazil \\ ${ }^{\mathrm{c}}$ College of Veterinary Medicine, Cornell University, Ithaca, NY 14853-5786, United States
}

\section{A R T I C L E I N F O}

\section{Keywords:}

Enterobacteriaceae

Food-producing animal

Antibiotic resistance

Adhesion

Invasion

\begin{abstract}
A B S T R A C T
There is an increasing number of reports worldwide about multidrug resistance (MDR) with potential of ExPEC in commensal E. coli. The present study evaluated the potential ExPEC in selected 44 MDR E.coli isolates, collected from livestock. ExPEC isolates were characterized by analysis of five main groups of virulence genes (papA and/or papC, sfa and/or foc, afa and/or dra, kpsMT II and iutA). We also determined the increased virulence potential analyzing other 29 virulence genes, the epidemiology of these isolates. Additionally, fifteen ExPEC isolates were selected to evaluate the adhesion and invasion capacity in vitro using Caco- 2 cells. Based on the analysis of the five main virulence genes, 72.7\% (32/44) strains were classified as ExPEC. The presence of each gene was iutA 88.6\%, KpsMT II 70.4\%, papC 25\%, sfa/focDE 4.5\%; afa/draBC genes were not found. All E. coli isolates were classified into: phylogenetic groups A (34\%), B1 (10\%), B2 (20\%), and D (36\%). MLST revealed 7 different STs among isolates, including a new ST identified (ST5687). The in vitro assay in Caco-2 cells showed that all isolates were capable to adhere or invade the epithelial cells, although this occurred at variable levels. The ExPEC isolate LO122 reached similar levels of invasion to the positive control strain Salmonella Typhimurium LT2. These results showed that the apparently commensal microbiota of poultry harbors MDR ExPEC isolates with high adhesion and invasion potential.
\end{abstract}

\section{Introduction}

Escherichia coli is a dynamic bacterial species that comprises the gut microbiota of humans and domestic animals. Although these bacteria are often referred as commensal, an important group known as extraintestinal pathogenic Escherichia coli (ExPEC), carries specific virulence genes that allow ability to colonize and survive out of the intestinal environment and to causes extraintestinal disease (Smith et al. 2007). These bacteria are considered potentially pathogenic and the variable sites of infection or hosts define the group of ExPEC, such as uropathogenic E. coli (UPEC) causing urinary tract infection, neonatal meningitis E. coli (NMEC), and sepsis-associated $E$. coli (SEPEC) or the host such as avian-pathogenic $E$. coli (APEC), causing colibacillosis (Belanger et al. 2011). The number of hospital and community infections caused by ExPEC are increasing worldwide, leading to a public health problem, especially if antimicrobial resistance is also related (Pitout 2012). ExPEC can acquire different antimicrobial resistance genes, reducing the therapeutic options and increasing the survival capacity in the environment, which has currently led to an increased risk for public health (Aslam et al. 2014). Some virulence factors are common to ExPEC isolates from both animals and humans allowing these bacteria to colonize, invade and cause infections outside the gastrointestinal tract (Johnson and Stell 2000). A number of characterized virulence factors are often associated to specific ExPEC groups, such as temperature-sensitive hemagglutinin (Tsh) protein in APEC, K1 capsule in NMEC, and Secreted Autotransporter Toxin (Sat) in UPEC. This examples show evidence that the acquisition and expression of certain genetic backgrounds are required for virulence. An important virulence factor is the production of adhesins, which might help them to adapt to different host tissues (Antao et al. 2009), but some adhesins are not specific to ExPEC, such as type I fimbriae (fimH) that can be present in 95\% of the non-harmfull E. coli (Tchesnokova et al. 2011).

Studies have found similarities among virulence genes in ExPEC isolates from humans and chickens, therefore poultry derived foods have been suggested as sources of ExPEC that causes infections in humans (Bergeron et al. 2012; Johnson et al. 2010; Manges and Johnson 2012). There are

\footnotetext{
* Corresponding author at: Departamento de Análises Clínicas, Toxicológicas e Bromatológicas, Faculdade de Ciências Farmacêuticas de Ribeirão Preto, Universidade de São Paulo (USP), Ribeirão Preto, SP 14040-903, Brazil.

E-mail address: aldarini@usp.br (A.L.C. Darini).
} 
different routes suggested that could lead to ExPEC and antimicrobial resistant isolates dissemination from animal to human environment, the most prominent are through meat consumption or environmental contamination by animal waste (Guenther et al. 2010). An important factor, contributing to the increased prevalence rates and dissemination of these isolates has been the intensive use of the antibiotics for treatments and prophylaxis in foodproducing animals. Despite antibiotics do not select virulent strains intrinsically (Johnson et al. 2004), virulence genes have often been reported in multidrug resistant (MDR) strains, facilitating the spread in diverse hosts (Guardabassi et al. 2004; Johnson et al. 2010). Infections by multidrug resistant isolates causes high economic impact to public health worldwide, increasing morbidity and mortality rates (Gastmeier et al. 2012), especially in developing countries with low investments in healthcare sector. Decades ago, the ExPEC were highly susceptible to antibiotics, but since the years 2000, these strains emerged with different resistance mechanisms to important classes of antibiotics, such as extended-spectrum cephalosporins and fluoroquinolones (Pitout 2012).

The present study evaluated the potential ExPEC profile in selected multidrug resistant E.coli isolates, collected from livestock. ExPEC isolates were characterized by analysis of five main groups of virulence genes including papA and/or papC (P fimbriae), sfa and/or foc (S and F1C fimbriae), afa and/or dra (binding and adhesion, respectively), kpsMT II (group II capsule syntesis), and iutA (aerobactin receptor). We also determined the increased virulence potential analyzing other 29 virulence genes and the epidemiology of these isolates. Additionally, fifteen ExPEC isolates were selected to evaluate the adhesion and invasion capacity in vitro using Caco- 2 cells as an intestinal epithelium model.

\section{Materials and methods}

\subsection{Strain selection and ExPEC classification}

Forty-four MDR E. coli isolates were selected from a previous characterized bacterial collection, harvested from apparently healthy poultry in Brazil in 2013 (Ferreira et al. 2016; Ferreira et al. 2017). Approved by the local Ethics Committee on the Use of Animals, protocol number 12.1.248.53.7. The selected isolates used in this study carried the following antimicrobial resistance genes: $b l a_{\mathrm{CTX}-\mathrm{M}-1}\left(1\right.$ isolate), $b l a_{\mathrm{CTX}-\mathrm{M}-2}(8$ isolates), $b l a_{\mathrm{CTX}-\mathrm{M}-8}\left(4\right.$ isolates) and $b l a_{\mathrm{CMY}-2}$ (31 isolates). The isolate was characterized as potential ExPEC if it harbored two or more of the five main groups of virulence genes (papA and/or papC, $s f a / f o c \mathrm{DE}, a f a / d r a \mathrm{BC}$, kpsM II and iutA) (Johnson and Stell 2000).

\subsection{Virulence genotyping}

For multiplex PCR studies screening for virulence genes, template DNA was prepared as previously described (Johnson and Stell 2000). The MDR E. coli isolates were tested for the presence of 34 virulence genes using specific primers in multiplex PCRs as previously described (Johnson and Stell 2000), including: hlyF (Johnson et al. 2006), iss, iroN, and ompT (Rodriguez-Siek et al. 2005). Moreover, positive controls were used for specific virulence genes. The $E$. coli isolates were characterized as potential ExPEC based on detection of at least 2 of the following 5 groups of ExPEC virulence genes: $p a p A$ and/or papC (P fimbriae), sfa and/or foc (S and F1C fimbriae), afa and/or dra (binding, adhesions respectively), kpsM II (group II capsule syntesis), and iutA (aerobactin receptor). (Johnson and Stell 2000; Johnson et al. 2009; Johnson et al. 2008b).

\subsection{Phylogenetic typing}

The isolates were assigned to phylogenetic groups according to the protocol previously described (Clermont et al. 2000). Based on this method, the isolates are classified into one of four groups (A, B1, B2, or D) determined by PCR.

\subsection{Multilocus sequence typing (MLST)}

MLST was carried out using the Achtman scheme (fumC, gyrB, icd, $m d h$, purA, recA, and adk genes) as previously described (Wirth et al. 2006). Alleles and sequence types (STs) were determined at EnteroBase, Escherichia coli MLST Database. (http://enterobase.warwick.ac.uk/ species/ecoli/allele_st_search).

\subsection{Caco-2 cells culture}

The human-derived intestinal epithelial Caco-2 cells, American Type Culture Collection, (ATCC, Manassas, VA, USA), were cultured in DMEM containing $4.5 \mathrm{~g} / 1$ glucose, $10 \%$ fetal bovine serum (FBS) (Sigma, USA), $2 \mathrm{mM}$ L-glutamine and $1 \%$ antibiotic-antimycotic solution, containing penicillin, streptomycin and amphotericin B (Life technologies, USA). Cells were incubated at $37^{\circ} \mathrm{C}$ and $5 \% \mathrm{CO}_{2}$, in $25 \mathrm{~cm}^{2}$ cell culture flasks (Corning, NY, USA) until form an adherent monolayer with $80 \%$ confluence, then washed with phosphate buffer saline (PBS, pH 7.4) and gently scrapped in the presence of trypsin $0.5 \%$. The cells were subsequently washed twice in PBS pH 7.4 and inoculated in 24 wells plate (Costar, Corning, NY, USA), using approximately $5 \times 10^{5}$ cells per well and maintained overnight in antibiotic-free DMEM media without FBS until bacterial infection. These cells were used for the in vitro adhesion and invasion assays with ExPEC MDR isolates.

\subsection{Invasion and adhesion assays in Caco-2 cells}

\subsubsection{Bacterial suspensions}

Fifteen ExPEC isolates were used in this study, selected based on the analysis of the greater number of virulence genes present and different sequence type (Table 1 ). Overnight bacterial cultures of selected isolates (Table 1), grown at $37^{\circ} \mathrm{C}$ in Luria-Bertani (LB) broth, were centrifuged at $1,650 \times g$ for $10 \mathrm{~min}$. The bacterial pellet was washed twice in PBS and diluted in pure DMEM media. The optical density (OD) for each isolate was measured in the spectrophotometer (Unicam UV8625, Thermo Scientific, USA) at $600 \mathrm{~nm}$ and adjusted to a concentration of $10^{8}$ colony forming units per $\mathrm{mL}(\mathrm{CFU} / \mathrm{mL})$. Caco- 2 cells were infected with ExPEC at a multiplicity of infection (MOI) of 100:1, inocula consisted of $10^{7} \mathrm{CFU}$ of bacteria in $100 \mathrm{uL}$ of DMEM (Darfeuille-Michaud et al. 2004).

\subsubsection{Measurement of the invasion}

The experiments were performed in triplicate in three different moments for all ExPEC isolates. The invasion assay and adhesion assay were always performed simultaneously. $10^{5}$ Caco-2 cells in each well were infected with the single ExPEC isolate and incubated for $90 \mathrm{~min}$ at $37^{\circ} \mathrm{C}$ and $5 \% \mathrm{CO} 2$. Following infection, cells were treated with freshly prepared meropenem solution $(80 \mu \mathrm{g} / \mathrm{ml})$ for $1 \mathrm{~h}$ to kill extracellular bacteria in each well (modified gentamycin protection assay). After treatment, cells were washed twice in sterile PBS, permeabilized in $0.1 \%$ Triton $\mathrm{X}-100$ for $30 \mathrm{~min}$. The infected cells were decimally diluted (from $10^{-1}$ to $10^{-3}$ ), plated on LB agar and incubated at $37^{\circ} \mathrm{C}$ for $18 \mathrm{~h}$ to calculate the number of invasive bacteria (intracellular). The invasive Salmonella Typhimurium LT2 strain (Accession NC_003197) was used as positive control and the sterility control used was the Caco-2 cells without inoculum.

\subsubsection{Measurement of the adhesion}

The assay was performed following previously described method. (Cieza et al. 2015; Frommel et al. 2013). The bacteria were suspended in DMEM in the presence of $2 \%$ D-mannose (Sigma), to block the E. coli adherence mechanism by type I pili. The plate was incubated for $3 \mathrm{~h}$ at $37^{\circ} \mathrm{C}$, after this period the Caco-2 cells were gently washed six times with sterile PBS to remove the non-adherent bacteria and lysed with $0.1 \%$ Triton X-100 for $30 \mathrm{~min}$. After lysis, the cell suspension was 
Table 1

Genotypic characteristics of the MDR E. coli isolates from chickens.

\begin{tabular}{|c|c|c|c|c|}
\hline Isolates & Virulence genes & Phylogenetic group & ExPEC & ST \\
\hline LO $132(*)$ & fimH,papEF,papC,papGI,papGII,iutA,kpsMTKI,kpsMTII,kpsMTK5,traT,ompT, hlyF & $\mathrm{D}$ & Yes & 93 \\
\hline LO $166^{\mathrm{a}}$ & fimH,papEF,papC,papGI,papGII,iutA,kpsMTKI,kpsMTII, kpsMTK5,traT,ompT,hlyF & A & Yes & 5687 \\
\hline LO $188(*)$ & fimH,papEF,papC,papGI,pap GII,iutA,kpsMTKI,kpsMTII,kpsMTK5,traT,ompT,hlyF & A & Yes & 5687 \\
\hline LO 146 & fimH,papEF,papC,papGI,papGII,iutA,kpsMTKI,kpsMTII,kpsMTK5,traT,ompT & A & Yes & 93 \\
\hline LO $167(*)$ & fimH,papEF,papC,papGI,papGII,iutA, kpsMTII,kpsMTK5,traT,ompT,hlyF & $\mathrm{D}$ & Yes & 5687 \\
\hline MA 09b $(*)$ & fimH,papEF,papC,papGI,papGII,papGIII,iutA,kpsMTK1,kpsMTII,traT & A & Yes & 93 \\
\hline LO $105($ ( ) & fimH,papEF,papC,papGI,papGII,iutA,kpsMTII,kpsMTK5,traT,ompT & A & Yes & 93 \\
\hline LO $129(*)$ & fimH,papEF,papC,papGI,papGII,iutA,kpsMTKI,kpsMTII,kpsMTK5,ompT & A & Yes & 93 \\
\hline LO 192 & fimH,papEF,papC,papGI,papGII,iutA,kpsMTKI,kpsMTII,kpsMTK5 & A & Yes & 93 \\
\hline LO $106(*)$ & fimH,ibeA,PAI,fyuA,iutA, kpsMTII,kpsMTK5,traT,hlyF & $\mathrm{D}$ & Yes & 68 \\
\hline LO 121b & fimH,fyuA,iutA,kpsMTKI,kpsMTII,kpsMTK5,traT,ompT,hlyF & B2 & Yes & 2309 \\
\hline LO $122(*)$ & fimH,fyuA,iutA,kpsMTKI,kpsMTII,kpsMTK5,traT,ompT,hlyF, & $\mathrm{D}$ & Yes & 2309 \\
\hline LO $127(*)$ & fimH,fyuA,iutA,kpsMTKI,kpsMTII,kpsMTK5,traT,ompT,hlyF & $\mathrm{D}$ & Yes & 2309 \\
\hline MA 04a $(*)$ & fimH,fyuA,iutA,kpsMTK1,kpsMTII,kpsMTK5,traT,ompT & $\mathrm{D}$ & Yes & 2309 \\
\hline LO 115 & fimH,fyuA, iutA, kpsMTII,kpsMTK5,traT, ompT, hlyF & $\mathrm{D}$ & Yes & 2309 \\
\hline LO $118(*)$ & fimH,papEF,papC,papGI,papGII,iutA,kpsMTKI,kpsMTII & A & Yes & 93 \\
\hline LO $164(*)$ & fimH,papEF,papC,papGI,papGII,iutA,kpsMTKI,kpsMTII & A & Yes & 93 \\
\hline MA $10 \mathrm{~b}$ & fimH,sfa/focDE,iutA, cvaC,traT,iss, iroN & B1 & Yes & nd \\
\hline MA 78 & fimH,iutA, cvaC,traT, iss, iroN,hlyF & B1 & No & - \\
\hline MA 1 & fimH,fyuA,sfa/focDE, iss, iroN & B1 & No & - \\
\hline MA 27 & fimH,iutA, kpsMTK1,kpsMTII,kpsMTK5 & B2 & Yes & 93 \\
\hline MA $36(*)$ & fimH,ibeA,iutA, kpsMTII,kpsMTK5 & $\mathrm{D}$ & Yes & 354 \\
\hline MA 57 & fimH,iutA, traT,ompT,hlyF & $\mathrm{D}$ & No & - \\
\hline MA 64 & fimH,iutA, traT,ompT, hlyF & $\mathrm{D}$ & No & - \\
\hline MA 75a & fimH,iutA, traT,ompT,hlyF & $\mathrm{D}$ & No & - \\
\hline MA 91 & fimH,iutA, kpsMTII,kpsMTK5,ompT & A & Yes & nd \\
\hline LO 101 & fimH,iutA, kpsMTKI,kpsMTII,kpsMTK5 & $\mathrm{B} 2$ & Yes & 93 \\
\hline LO $126 \mathrm{a}(*)$ & fimH,ibeA,iutA,kpsMTII,kpsMTII & $\mathrm{D}$ & Yes & 354 \\
\hline LO 128 & fimH,iutA, kpsMTKI,kpsMTII,kpsMTK5 & B2 & Yes & 93 \\
\hline LO 131 & fimH,iutA, kpsMTKI,kpsMTII,kpsMTK5 & B2 & Yes & 93 \\
\hline LO 139 & fimH,iutA, traT,ompT,hlyF & $\mathrm{D}$ & No & - \\
\hline LO 175 & fimH,ibeA,iutA,kpsMTKI,kpsMTII, & $\mathrm{D}$ & Yes & nd \\
\hline LO $180(*)$ & fimH,iutA, kpsMTII, $k p s M T K 5$, ompT & A & Yes & 93 \\
\hline LO 183 & fimH,iutA, kpsMTII, $k p s M T K 5$, ompT & A & Yes & nd \\
\hline MA 52 & fimH,iutA, $k p s M T I I, k p s M T K 5$ & $\mathrm{~B} 2$ & Yes & 93 \\
\hline MA 85 & fimH,iutA,traT,ompT & $\mathrm{D}$ & No & - \\
\hline MA 94 & fimH,fyuA,papGIII,ompT & A & No & 2197 \\
\hline MA 97 & fimH,iutA,traT,ompT & $\mathrm{D}$ & No & - \\
\hline LO 117a & fimH,iutA,kpsMTII,kpsMTK5 & $\mathrm{B} 2$ & Yes & 93 \\
\hline LO 134 & fimH,iutA,kpsMTII,kpsMTK5 & B2 & Yes & 93 \\
\hline LO 140 & fimH,iutA,kpsMTII,kpsMTK5 & $\mathrm{B} 2$ & Yes & 93 \\
\hline MA 12 & fimH,traT,ompT & A & No & - \\
\hline MA 49a & fimH,traT,hlyF & B1 & No & - \\
\hline MA 18a & fimH & $\mathrm{D}$ & No & 1011 \\
\hline
\end{tabular}

* Isolates included in adhesion/invasion assay; nd: not determined; ( $)$ : not performed. The main virulence genes that characterized as ExPEC are typed with bold.

inoculated pure and decimally diluted $\left(10^{-1}\right.$ to $\left.10^{-3}\right)$ in LB agar plates, incubated for $18 \mathrm{~h}$ at $37 \mathrm{C}$. The number of CFU were counted on the agar plates to obtain the total bacterial number (invasive and adherent). Posteriorly, the numbers of invasive bacteria were subtracted, to calculate the number of adherent bacteria. The statistical differences in the bacterial numbers found after adhesion and invasion assay were determined by the analysis of variance (ANOVA) with Tukey test using GraphPad Prism software (GraphPad, USA). The limit of significance considered was $p<.05$.

\subsection{Confocal microscopy}

Confluent monolayers of Caco-2 cells were infected with ExPEC (MOI of 100:1) in Chamber Slide ${ }^{\mathrm{TM}}$ System (Nunc ${ }^{\mathrm{TM}}$ Lab-Tek $^{\mathrm{TM}} \mathrm{II}$ ) for $3 \mathrm{~h}$ at $37 \mathrm{C}$ and $5 \% \mathrm{CO}_{2}$ cells, and gently washed three times with sterile $\mathrm{PBS} \mathrm{pH} 7.4$ to remove non-adherent bacteria. Cells were fixed with $4 \%$ paraformaldehyde in sterile PBS at $4{ }^{\circ} \mathrm{C}$, overnight. Before analysis, cells were washed three times with sterile PBS. The ExPEC isolates were stained with fluorophore propidium iodide solution (P4864, Sigma-Aldrich, USA) and Caco-2 cells were visualized at light microscope. The slides were analyzed, photographed and overlaid on the confocal microscope Leica TCS SP8 (Leica Microsystems, USA).

\section{Results}

\subsection{Prevalence of virulence factors and ExPEC isolates}

After the analyses of the virulence genes in 44 strains, the presence were of $100 \%$ fimH, 88.6\% iutA, 70.4\% KpsMT II, 59\% KpsMT (K5), $52.2 \%$ oтpT, $47.7 \%$ traT, 38.6\% KpsMT(K1), 34\% hlyF, 25\% papC, papEF, papGI and papGII, 18\% fyuA, 9\% ibeA, 6.8\% iroN and iss, $4.5 \%$ sfa/focDE and $c v a C, 2.3 \%$ PAI and papGIII. The genes papAH, KpsMT III, bmaE, hlyA, rfc, nfaE, gafD, cdtB, focG, afa/draBC, cnf1 and sfaS were not detected in the isolates.

As shown $32 / 44(72.7 \%)$ of the isolates were classified as ExPEC based on the criteria established previously and $27.3 \%$ were defined as non-ExPEC (Table 1).

The 15 isolates selected to perform the invasion and adhesion assays were $E$. coli MA(04,09,36), LO(105,106,118,122,126a,127,129,132, $164,167,180,188$ ) based on the presence of important virulence genes.

\subsection{Phylogenetic groups}

The 44 E. coli isolates were classified into phylogenetic groups A (34\%), B1 (10\%), B2 (20\%), and D (36\%). Considering the 32 potential ExPEC isolates, $38 \%$ belonged to group A, $28 \%$ belonged to group 
groups B2, 31\% were classified in group D and 3\% were group B1. All isolates of the group B2 were classified as ExPEC whereas, among the ExPEC isolates, group A was the most prevalent (Table 1).

\subsection{Multi locus sequence typing (MLST)}

MLST revealed 7 different STs (Table 1), which 6 were previously reported in the database. Alleles and ST that had not been previously described were assigned new designations by the curators of the database. One new ST (ST 5687) was characterized in three $E$. coli isolates. Among the 7 STs identified in this study, four (ST68, 93, 354,1011) were previously reported in ExPEC isolates in other countries, all associated with avian infection (APEC) and urinary tract infection (UPEC) (http://mlst.ucc.ie/mlst/dbs/Ecoli) (Table 1).

\subsection{Adhesion assay}

A variable adhesion capacity was observed, after the quantification of extracellular bacteria (Fig. 1). The isolates MA04 (fimH), MA36 (fimH), LO106 (fimH), LO126a (fimH), LO127 (fimH) LO132 (fimH, papC, papEF, pap GI, GII and GIII), LO164 (fimH, papC, papEF, pap GI, GII and GIII) and LO167 (fimH, papC, papEF, pap GI, GII and GIII) showed a higher capacity of adherence.

Isolates 105 and LO118 (fimH, papEF, papC, papGI and GII) showed the lowest adhesion capacity. Isolates 122 (fimH), LO129 (fimH, papC, papEF, papGI, GII and GIII), LO180 (fimH) and LO188 (fimH, papC, papEF, papGI, GII and GIII) showed low adherence levels at the evaluated moment due to their high invasion capacity.

\subsection{Invasion assay}

All isolates included in adhesion assays were also tested for the invasion capacity in Caco- 2 cells. The invasion potential of isolate LO122 was significantly higher, demonstrating similar levels of invasion to positive control strain Salmonella Typhimurium LT2 $(p>.05)$. The isolates LO129, LO167, LO180 and LO188 also showed higher rate of invasion $(\mathrm{p}>.05)$. The isolates.

MA04, MA09, LO118 and LO164 showed a significantly lower number of invasive bacteria in Caco-2 cells (Fig. 1).

\subsection{Confocal microscopy}

Fig. 2 shows the confocal microscopy analysis of some isolates to

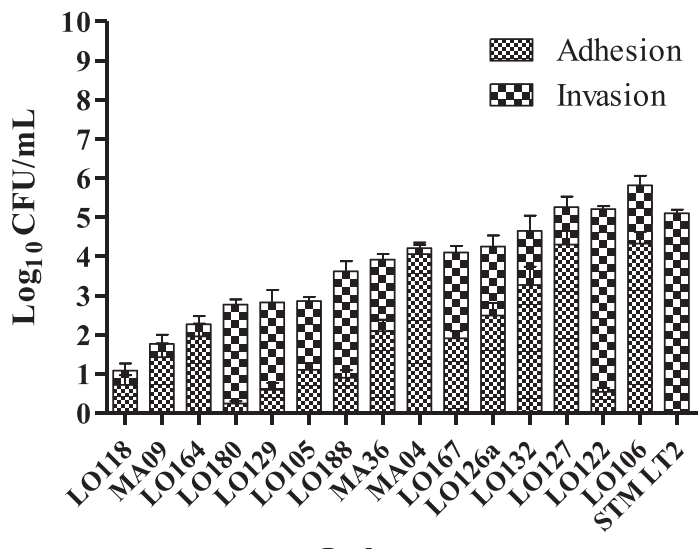

\section{Isolates}

Fig. 1. Quantitative analysis of adherence and invasion in Caco-2 cells infected with potential ExPEC isolates. Values were considered significantly different at $p<.05$. Standard deviations (SD) values are represented by error bars and represent the average quantification values obtained from the three repetitions. STM: Salmonella Typhimurium LT2. evaluate the phenotype obtained in adhesion and invasion assay. It is possible to visualize the high invasiveness of control strain Salmonella Typhimurium LT2 and a similar profile was observed only for isolate LO122. ExPEC isolate MA04, demonstrates high adhesion capacity and isolate LO118 represents the profile of an isolate with low adherence and invasion capacity.

\section{Discussion}

In this study, we characterized the presence of the virulence genes in a collection of ESBL- and AmpC-producing E. coli isolates from commercial chickens previously reported as MDR bacteria (Ferreira et al. 2016). The use of antimicrobials does not lead to the selection of virulent strains, but rather to the spread and maintenance of resistance genes among commensal strains (Johnson et al. 2010). The increase of MDR in the commensal microbiota of poultry may have contributed to emergence of MDR ExPEC in infections in humans (Pitout 2012). An increased ESBL- and AmpC-producing ExPEC isolates has been reported in human infections in Canadian hospitals (Denisuik et al. 2013).

The emergence of ESBL genes in poultry may be associated with the prophylactic use of cephalosporins injected into eggs to prevent diseases in newly hatched chicks (Dutil et al. 2010). In Brazil, third-generation cephalosporins have been associated with in ovo vaccination at 18th incubation day, creating a homogeneous condition for the selection of MDR microbiota in a massive animal population.

The association of multidrug resistance with pathogenic bacteria, such as ExPEC and Salmonella, increases the impact these bacteria may cause to animal and human health. Most of our isolates showed the genetic load of ExPEC, however these bacteria were isolated from apparently healthy poultry, intended for slaughter and consumption. Studies about the zoonotic risk of ExPEC have reported the concern to public health in cases where transmission occurs through the food chain (Bergeron et al. 2012; Vincent et al. 2010). The ExPEC are present in the intestinal microbiota of a fraction of the healthy population and asymptomatically colonize the gut. However, these bacteria are able to survive in extra intestinal environments and cause disease in humans and animals (Clermont et al., 2011; Ranjan et al., 2017). The gene iutA promotes increased capacity to colonize the humans and was found in $>80 \%$ of the isolates studied in this work. This gene was also the most identified in $E$. coli isolated from commercial chicken carcasses in Brazil (Koga et al. 2015). The gene KpsMT II (polysaccharide capsule marker genes) was found in $70 \%$ of the isolates studied. This gene has been more frequently reported in ExPEC isolates from humans than from poultry, however our data suggests this virulence gene may also reach a high prevalence within poultry farms (Belanger et al. 2011). Epidemiological studies suggested that poultry may act as source of ExPEC for humans, in cases where carcass contamination is high. Genetic similarities between isolates from both healthy poultry and processed poultry meat, have evidenced the dissemination of these bacteria through the food chain (Manges and Johnson 2012).

Genes involved in bacterial serum resistance are essential for development of bacteremia. In our study $47 \%$ of the ExPEC isolates carried this gene. The results obtained from this work showed that the apparently commensal $E$. coli isolates contained many virulence factors associated with colonization and persistence of the isolate thereby increasing the potential to cause disease in poultry and humans. In Brazil, E. coli isolates from poultry carcasses isolated between 2007 and 2013, showed an increased level of antibiotic resistance and virulence (associated with APEC) in more recent isolates in comparison with isolates from 2007 (Koga et al. 2015).

In the present work, the four phylogenetic groups were found and group D was the most predominant. ExPEC isolates have been mainly classified into group B2 and to a lesser extent into group D, while intestinal commensal isolates belongs to groups A and B1 (Clermont et al. 

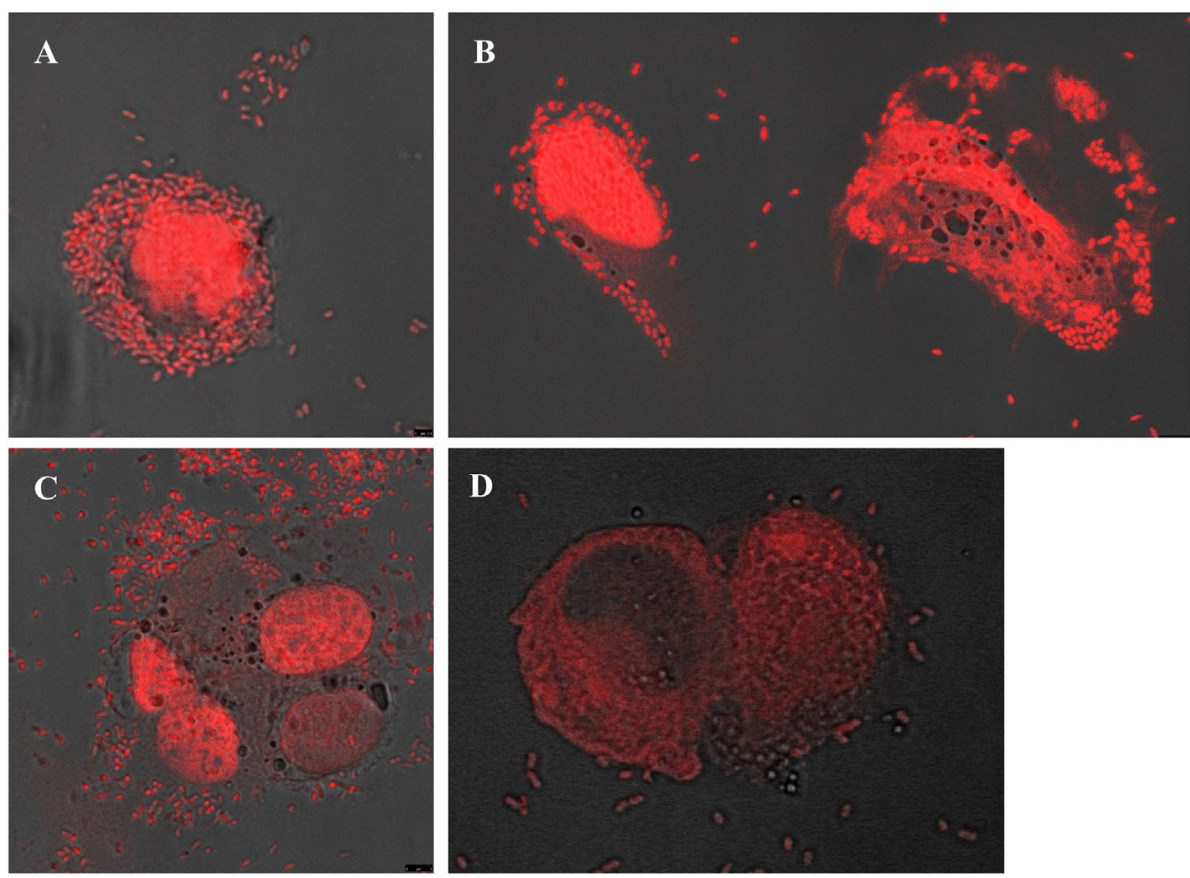

Fig. 2. Confocal images of in vitro evaluation of adhesion and invasion of MDR E. coli isolates and Salmonella Typhimurium LT2 (stained by propridium iodide, in red) in Caco-2 cells. (A) Salmonella Typhimurium LT2 used as an invasive control strain. (B) Phenotypic profile of a highly invasive MDR $E$. coli isolate with ExPEC potential reaching high numbers of intracellular bacteria after infection (isolate LO122). (C) Phenotypic profile of an adherent MDR E.coli isolate with ExPEC potential demonstrating the strong capacity to attach the cell surface after infection (isolate MA04). (D) Phenotypic profile of an MDR E.coli isolate carrying ExPEC genes, with with low adherence and invasion capacity (isolate LO118). (For interpretation of the references to colour in this figure legend, the reader is referred to the web version of this article.)
2000; Johnson et al. 2008a). Our results revealed that the phylogenetic group D and phylogenetic group B2 were found in most isolates, however the isolates were obtained from apparently healthy poultry and they are considered commensal, once these were not causing extraintestinal infections during the sampling. The asymptomatic gut carriage of B2 phylogroup has a variable prevalence. This is based on epidemiological factors, such as the geographical area, population analyzed and origin of samples. Virulence factors associated to ExPEC may belong to any phylogroup. The literature demonstrated that in a large proportion of healthy community, the intestinal colonization by ExPEC among other commensal Enterobacteriaceae, may act as a potential reservoir for the pathogenic variants (Erjavec and Zgur-Bertok 2015).

The ST93, was first identified in Europe and since 1999 has been associated to ExPEC isolates. In Brazil, ST93 was reported in 2001 and 2008 associated to human infections in the northeast region of the country and the isolates were characterized as ExPEC (http://mlst.ucc. ie/dbs/Ecoli). The ST93 was also found in poultry (APEC) and in humans (ExPEC) causing infections (Berman et al. 2014; Chen et al. 2013; Dierikx et al. 2013; Maluta et al. 2014). The ST354 was reported in Brazil in 2005 and 2009 associated to human infections caused by ExPEC in two different regions of the country (http://mlst.ucc.ie/dbs/ Ecoli). In Europe, ST354 was also found worldwide associated to ExPEC (Guo et al. 2015; Mora et al. 2011). The ST68 was isolated in Asia in ExPEC isolates from humans (A. et al., 2017), but this ST had never been reported in Brazil.

ExPEC produce a wide variety of adhesins, which might help to colonize different host tissues (Antao et al. 2009). In the present study, variable levels of adherence to Caco- 2 cells were observed in vitro, among the 15 isolates phenotypically evaluated (Fig. 1). Quantitative assessment of invasive bacteria was performed by meropenem protection assay (modified gentamycin protection assay) and results obtained in this assay were similar to those observed in confocal microscopy evaluation, as demonstrated in Fig. 2.

As demonstrated in Fig. 1, five ExPEC isolates MA36, LO105, LO118, LO126a and LO167 showed a balanced capacity between adhesion and invasion. However, other isolates showed a profile with higher ability to rather to adhere or to invade the cells. Most prominent profiles of invasion, were noticed in isolates LO122, LO129, LO180 and LO188, where this isolates were found in high numbers intracellularly, however, considerably low numbers of bacteria were adhered at the evaluation moment. Remarkably, LO122 showed invasion levels similar to the invasive control strain Salmonella Typhimurium LT2. Isolates LO106, LO127 and LO132 demonstrate the phenotype of isolates which were more adherent than invasive. Confocal microscopy confirmed the phenotype of these isolates and as shown in Fig. 2, isolate LO122, was highly invasive and was found in high numbers in the cytoplasm of Caco- 2 cells after $3 \mathrm{~h}$ of infection.

Adhesins, as produced by gene fim $\mathrm{H}$ present in all isolates, have been proposed as the first necessary step for colonization, furthermore it has the ability to act different steps of the infection process (Tchesnokova et al. 2011). Type 1 fimbriae are produced by isolates of E. coli, being an important virulence determinant involved in adhesion in cells (Antao et al. 2009). The pap gene cluster has 11 genes, the papF and $p a p G$ encoding proteins are essential for adhesion and the product of the papC being required for biogenesis (Kuehn et al. 1992). The animal isolates evaluated, carried pap genes (25\%) and fim $\mathrm{H}(100 \%)$.

In Brazil, around 58\% of ExPEC resistant to antimicrobials isolated from humans were reported as adherent to Caco-2 cells (CergoleNovella et al. 2015). In the present study, among the 15 MDR E. coli phenotypically analyzed in vitro, $100 \%$ showed ability to adhesion in Caco-2 cells. However, isolates with higher invasion capacity, such as LO122, demonstrated a lower number of adherent bacteria. Adhesion mechanism is necessary for intracellular infection, although, these highly invasive isolates may adhere for a short time before invasion.

The use of antimicrobials does not lead to the selection of virulent strains (Johnson et al. 2004) but rather to the spread of resistance genes among commensal strains. All 44 isolates selected were determined as MDR and around $72 \%$ showed potential to become ExPEC. The antimicrobial resistance is a constant threat to public health worldwide and this concern increase when these isolates display many virulence factors increasing the pathogenic potential. Foodborne infections with ExPEC, resistant to important antimicrobials, have been a concern to the public health. In this study, $E$. coli isolates from poultry microbiota carrying many virulence genes, characterized this commensal bacteria as potential ExPEC, withadhesion and invasion ability. These factors associated to multiresistance to antibiotics showed the necessity to reducethe use of antibiotics in poultry industry and frequently perform the monitoring of $E$. coli in the microbiota of food producing animals. 


\section{Conflict of interest statement}

\section{None to declare.}

\section{Acknowledgements}

We would like to thank DVM, Mark Ishi, who contributed for sampling in poultry Farms, Dr. Luke Richards for his kind review of the text and São Paulo Research Foundation (FAPESP) for the constant support for our research (Grant n. 2014/14494-8). Rafael. Penha Filho was funded by São Paulo Research Foundation (FAPESP, grant 2017/ 00620-0), L.N.A. was supported by post-doctoral fellowship, from National Council for Scientific and Technological Development (CNPq/ PNPD/2017).

\section{References}

Antao, E.M., Wieler, L.H., Ewers, C., 2009. Adhesive threads of extraintestinal pathogenic Escherichia coli. Gut Pathog. 1, 22.

Aslam, M., Toufeer, M., Narvaez Bravo, C., Lai, V., Rempel, H., Manges, A., Diarra, M.S., 2014. Characterization of Extraintestinal pathogenic Escherichia coli isolated from retail poultry meats from Alberta. Canada. Int. J. Food Microbiol. 177, 49-56.

Belanger, L., Garenaux, A., Harel, J., Boulianne, M., Nadeau, E., Dozois, C.M., 2011. Escherichia coli from animal reservoirs as a potential source of human extraintestinal pathogenic E. coli. FEMS Immunol. Med. Microbiol. 62, 1-10.

Bergeron, C.R., Prussing, C., Boerlin, P., Daignault, D., Dutil, L., Reid-Smith, R.J., Zhanel, G.G., Manges, A.R., 2012. Chicken as reservoir for extraintestinal pathogenic Escherichia coli in humans. Canada. Emerg. Infect. Dis. 18, 415-421.

Berman, H., Barberino, M.G., Moreira Jr., E.D., Riley, L., Reis, J.N., 2014. Distribution of strain type and antimicrobial susceptibility of Escherichia coli isolates causing meningitis in a large urban setting in Brazil. J. Clin. Microbiol. 52, 1418-1422.

Cergole-Novella, M.C., Pignatari, A.C., Guth, B.E., 2015. Adhesion, biofilm and genotypic characteristics of antimicrobial resistant Escherichia coli isolates. Braz. J. Microbiol. 46, 167-171.

Chen, Y., Chen, X., Zheng, S., Yu, F., Kong, H., Yang, Q., Cui, D., Chen, N., Lou, B., Li, X., Tian, L., Yang, X., Xie, G., Dong, Y., Qin, Z., Han, D., Wang, Y., Zhang, W., Tang, Y.W., Li, L., 2013. Serotypes, genotypes and antimicrobial resistance patterns of human diarrhoeagenic Escherichia coli isolates circulating in southeastern China. Clin. Microbiol. Infect. 20, 52-58.

Cieza, R.J., Hu, J., Ross, B.N., Sbrana, E., Torres, A.G., 2015. The IbeA Invasin of adherent-invasive Escherichia coli mediates interaction with intestinal epithelia and macrophages. Infect. Immunity 83, 1904-1918.

Clermont, O., Bonacorsi, S., Bingen, E., 2000. Rapid and simple determination of the Escherichia coli phylogenetic group. Appl. Environ. Microbiol. 66, 4555-4558.

Clermont, O., Olier, M., Hoede, C., Diancourt, L., Brisse, S., Keroudean, M., Glodt, J., Picard, B., Oswald, E., Denamur, E., 2011. Animal and human pathogenic Escherichia coli strains share common genetic backgrounds. Infect. Genet. Evolut. 11, 654-662.

Darfeuille-Michaud, A., Boudeau, J., Bulois, P., Neut, C., Glasser, A.L., Barnich, N., Bringer, M.A., Swidsinski, A., Beaugerie, L., Colombel, J.F., 2004. High prevalence of adherent-invasive Escherichia coli associated with ileal mucosa in Crohn's disease. Gastroenterol. 127, 412-421.

Denisuik, A.J., Lagace-Wiens, P.R., Pitout, J.D., Mulvey, M.R., Simner, P.J., Tailor, F., Karlowsky, J.A., Hoban, D.J., Adam, H.J., Zhanel, G.G., 2013. Molecular epidemiology of extended-spectrum beta-lactamase-, AmpC beta-lactamase- and carbapenemase-producing Escherichia coli and Klebsiella pneumoniae isolated from Canadian hospitals over a 5 year period: CANWARD 2007-11. J. Antimicrob. Chemother. 68 (Suppl. 1), i57-i65.

Dierikx, C., van der Goot, J., Fabri, T., van Essen-Zandbergen, A., Smith, H., Mevius, D., 2013. Extended-spectrum-beta-lactamase- and AmpC-beta-lactamase-producing Escherichia coli in Dutch broilers and broiler farmers. J. Antimicrob. Chemother. 68, $60-67$.

Dutil, L., Irwin, R., Finley, R., Ng, L.K., Avery, B., Boerlin, P., Bourgault, A.M., Cole, L., Daignault, D., Desruisseau, A., Demczuk, W., Hoang, L., Horsman, G.B., Ismail, J., Jamieson, F., Maki, A., Pacagnella, A., Pillai, D.R., 2010. Ceftiofur resistance in Salmonella enterica serovar Heidelberg from chicken meat and humans. Canada. Emerg. Infect. Dis. 16, 48-54.

Erjavec, M.S., Zgur-Bertok, D., 2015. Virulence potential for extraintestinal infections among commensal Escherichia coli isolated from healthy humans-the Trojan horse within our gut. FEMS Microbiol. Lett. 362.

Ferreira, J.C., Penha Filho, R.A., Andrade, L.N., Berchieri Junior, A., Darini, A.L., 2016 Evaluation and characterization of plasmids carrying CTX-M genes in a non-clonal population of multidrug-resistant Enterobacteriaceae isolated from poultry in Brazil. Diagn. Microbiol. Infect. Dis. 85, 444-448.

Ferreira, J.C., Penha Filho, R.A.C., Andrade, L.N., Berchieri Junior, A., Darini, A.L.C. 2017. Diversity of plasmids harboring bla $a_{\mathrm{CMY}-2}$ in multidrug-resistant Escherichia coli isolated from poultry in Brazil. Diagn. Microbiol. Infect. Dis. 88, 361-364.

Frommel, U., Lehmann, W., Rodiger, S., Bohm, A., Nitschke, J., Weinreich, J., Gross, J., Roggenbuck, D., Zinke, O., Ansorge, H., Vogel, S., Klemm, P., Wex, T., Schroder, C., Wieler, L.H., Schierack, P., 2013. Adhesion of human and animal Escherichia coli strains in association with their virulence-associated genes and phylogenetic origins. Appl. Environ. Microbiol. 79, 5814-5829.

Gastmeier, P., Schwab, F., Meyer, E., Geffers, C., 2012. Excess mortality and prolongation of stay due to bloodstream infections caused by multiresistant pathogens in Germany. Dtsch. Med. Wochenschr. 137, 1689-1692.

Guardabassi, L., Schwarz, S., Lloyd, D.H., 2004. Pet animals as reservoirs of antimicrobial-resistant bacteria. J. Antimicrob. Chemother. 54, 321-332.

Guenther, S., Grobbel, M., Lubke-Becker, A., Goedecke, A., Friedrich, N.D., Wieler, L.H., Ewers, C., 2010. Antimicrobial resistance profiles of Escherichia coli from common European wild bird species. Vet. Microbiol. 144, 219-225.

Guo, S., Wakeham, D., Brouwers, H.J., Cobbold, R.N., Abraham, S., Mollinger, J.L., Johnson, J.R., Chapman, T.A., Gordon, D.M., Barrs, V.R., Trott, D.J., 2015. Humanassociated fluoroquinolone-resistant Escherichia coli clonal lineages, including ST354, isolated from canine feces and extraintestinal infections in Australia. Microbes Infect. 17, 266-274.

Johnson, J.R., Stell, A.L., 2000. Extended virulence genotypes of Escherichia coli strains from patients with urosepsis in relation to phylogeny and host compromise. J. Infect. Dis. 181, 261-272.

Johnson, J.R., Kuskowski, M.A., Gajewski, A., Sahm, D.F., Karlowsky, J.A., 2004. Virulence characteristics and phylogenetic background of multidrug-resistant and antimicrobial-susceptible clinical isolates of Escherichia coli from across the United States, 2000-2001. J. Infect. Dis. 190, 1739-1744.

Johnson, T.J., Siek, K.E., Johnson, S.J., Nolan, L.K., 2006. DNA sequence of a ColV plasmid and prevalence of selected plasmid-encoded virulence genes among avian Escherichia coli strains. J. Bacteriol. 188, 745-758.

Johnson, T.J., Wannemuehler, Y., Doetkott, C., Johnson, S.J., Rosenberger, S.C., Nolan, L.K., 2008a. Identification of minimal predictors of avian pathogenic Escherichia coli virulence for use as a rapid diagnostic tool. J. Clin. Microbiol. 46, 3987-3996.

Johnson, T.J., Wannemuehler, Y., Johnson, S.J., Stell, A.L., Doetkott, C., Johnson, J.R., Kim, K.S., Spanjaard, L., Nolan, L.K., 2008b. Comparison of extraintestinal pathogenic Escherichia coli strains from human and avian sources reveals a mixed subset representing potential zoonotic pathogens. Appl. Environ. Microbiol. 74, 7043-7050.

Johnson, T.J., Logue, C.M., Wannemuehler, Y., Kariyawasam, S., Doetkott, C., Debroy, C., White, D.G., Nolan, L.K., 2009. Examination of the source and extended virulence genotypes of Escherichia coli contaminating retail poultry meat. Foodborne Pathog. Dis. 6, 657-667.

Johnson, T.J., Jordan, D., Kariyawasam, S., Stell, A.L., Bell, N.P., Wannemuehler, Y.M., Alarcon, C.F., Li, G., Tivendale, K.A., Logue, C.M., Nolan, L.K., 2010. Sequence analysis and characterization of a transferable hybrid plasmid encoding multidrug resistance and enabling zoonotic potential for extraintestinal Escherichia coli. Infect. Immunity 78, 1931-1942.

Koga, V.L., Rodrigues, G.R., Scandorieiro, S., Vespero, E.C., Oba, A., de Brito, B.G., de Brito, K.C., Nakazato, G., Kobayashi, R.K., 2015. Evaluation of the antibiotic resistance and virulence of Escherichia coli strains isolated from chicken carcasses in 2007 and 2013 from Parana. Brazil. Foodborne Pathog. Dis. 12, 479-485.

Kuehn, M.J., Heuser, J., Normark, S., Hultgren, S.J., 1992. P pili in uropathogenic E. coli are composite fibres with distinct fibrillar adhesive tips. Nature 356, 252-255.

Maluta, R.P., Logue, C.M., Casas, M.R., Meng, T., Guastalli, E.A., Rojas, T.C., Montelli, A.C., Sadatsune, T., de Carvalho Ramos, M., Nolan, L.K., da Silveira, W.D., 2014. Overlapped sequence types (STs) and serogroups of avian pathogenic (APEC) and human extra-intestinal pathogenic (ExPEC) Escherichia coli isolated in Brazil. PLoS One 9, e105016.

Manges, A.R., Johnson, J.R., 2012. Food-borne origins of Escherichia coli causing extraintestinal infections. Clin. Infect. Dis. 55, 712-719.

Mora, A., Blanco, M., Lopez, C., Mamani, R., Blanco, J.E., Alonso, M.P., Garcia-Garrote, F., Dahbi, G., Herrera, A., Fernandez, A., Fernandez, B., Agulla, A., Bou, G., Blanco, J., 2011. Emergence of clonal groups O1:HNM-D-ST59, O15:H1-D-ST393, O20:H34/ HNM-D-ST354, O25b:H4-B2-ST131 and ONT:H21,42-B1-ST101 among CTX-M-14producing Escherichia coli clinical isolates in Galicia, northwest Spain. Int. J.Antimicrob. Agents 37, 16-21.

Pitout, J.D., 2012. Extraintestinal pathogenic Escherichia coli: a combination of virulence with antibiotic resistance. Frontiers Microbiol. 3, 9.

Ranjan, A., Shaik, S., Nandanwa, N., Hussain, A., Tiwari, K.S., Semmler, T., Jadhav, S., Weiler, H.L., Alam, M., Cowell, R.R., Ahemed, N., 2017. Comparative genomics of Escherichia coli Isolated from skin and soft tissue and other extraintestinal infections. mBio 8.

Rodriguez-Siek, K.E., Giddings, C.W., Doetkott, C., Johnson, T.J., Nolan, L.K., 2005. Characterizing the APEC pathotype. Vet. Res. 36, 241-256.

Smith, J.L., Fratamico, P.M., Gunther, N.W., 2007. Extraintestinal pathogenic Escherichia coli. Foodborne Pathog. Dis. 4, 134-163.

Tchesnokova, V., Aprikian, P., Kisiela, D., Gowey, S., Korotkova, N., Thomas, W., Sokurenko, E., 2011. Type 1 fimbrial adhesin FimH elicits an immune response that enhances cell adhesion of Escherichia coli. Infect. Immunity 79, 3895-3904.

Vincent, C., Boerlin, P., Daignault, D., Dozois, C.M., Dutil, L., Galanakis, C., Reid-Smith, R.J., Tellier, P.P., Tellis, P.A., Ziebell, K., Manges, A.R., 2010. Food reservoir for Escherichia coli causing urinary tract infections. Emerg. Infect. Dis. 16, 88-95.

Wirth, T., Falush, D., Lan, R., Colles, F., Mensa, P., Wieler, L.H., Karch, H., Reeves, P.R., Maiden, M.C., Ochman, H., Achtman, M., 2006. Sex and virulence in Escherichia coli: an evolutionary perspective. Mol. Microbiol. 60, 1136-1151. 